\title{
Registry-based randomized clinical trials as a method to improve cancer care in Australia
}

Siavash Foroughi, ${ }^{1,2}$ Hui-li Wong ${ }^{1,2,3}$, Lucy Gately ${ }^{1,2}$, Margaret Lee ${ }^{1,2,4,5}$, Koen Simons ${ }^{6,7}$, Jeanne Tie ${ }^{1,2,3,5}$, Antony Wilks Burgess ${ }^{2,8,9}$, Peter Gibbs ${ }^{1,2,5}$

${ }^{1}$ Systems Biology and Personalised Medicine Division, The Walter and Eliza Hall Institute of Medical Research, Parkville, Victoria, Australia

${ }^{2}$ Department of Medical Biology, The University of Melbourne, Parkville, Victoria, Australia

${ }^{3}$ Department of Medical Oncology, Peter MacCallum Cancer Centre, Melbourne, Victoria, Australia

${ }^{4}$ Department of Medical Oncology, Eastern Health, Box Hill, Victoria, Australia

${ }^{5}$ Department of Medical Oncology, Western Health, St Albans, Victoria, Australia

${ }^{6}$ Centre for Epidemiology and Biostatistics, Melbourne School of Population and Global Health, The University of Melbourne, Parkville, Victoria, Australia

${ }^{7}$ Western Centre for Health, Research and Education, Western Health, St Albans, Victoria, Australia

${ }^{8}$ Department of Surgery, Royal Melbourne Hospital, The University of Melbourne, Parkville, Victoria, Australia

${ }^{9}$ Structural Biology Division, The Walter and Eliza Hall Institute of Medical Research, Parkville, Victoria, Australia

Running title: Registry-based trials

Corresponding author:

Mr Siavash Foroughi

Systems Biology and Personalised Medicine Division

The Walter and Eliza Hall Institute of Medical Research

1 G Royal Parade, Parkville VIC 3052, Australia

Tel: +61393452894

Email: foroughi@,wehi.edu.au

Keywords: registries , research design , registry-based randomized clinical trial

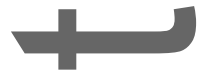

We thank Tang et al. ${ }^{1}$ for endorsing our efforts to establish registry-based randomized clinical trials (RRCTs) to improve cancer care. ${ }^{2}$ They raise some issues that are worthy of further discussion: especially, with respect to their summary Table where they describe differences between conventional randomized clinical trials (RCTs), RRCTs and population-based observational research; this discussion also highlights some recent significant developments with our RRCT activity.

Regarding the "Strengths" listed in their summary Table, we believe it is important to note that the cost differential between conventional and RRCTs is substantial, simply stating that the costs of This is the author manuscript accepted for publication and has undergone full peer review but has not been through the copyediting, typesetting, pagination and proofreading process, which may lead to differences between this version and the Version of Record. Please cite this article as doi: 10.1111/ajco.13122.

This article is protected by copyright. All rights reserved. 
RRCTs are lower does not properly capture this differential. Conventional RCTs cost many thousands or tens of thousands of dollars per patient enrolled versus as little as fifty dollars per patient for an RRCT. ${ }^{3}$ The "Weaknesses" in their Table do not accurately capture the registry infrastructure that is supporting RRCTs. The registries being used, such as TRACC for metastatic colorectal cancer, which was established in $2009,{ }^{4}$ are long-standing registries so there is no need to establish or maintain a dedicated registry for the RRCT. There are many such resources that are already fully funded and capable of supporting a broad range of research activities. Using the current registry resources to capture treatment and outcome data for an RRCT adds further value to these existing activities.

We are colfecting data in comprehensive disease-based registries: capturing treatment and outcome data across multiple lines of therapy for specific disease types. These trials-oriented registries are distinctly different to traditional clinical quality registries that only capture limited data sets at the time of diagnosis. By using comprehensive disease registries, we believe there is no limit to the range of RRCT questions that can be explored effectively, whether they address treatment responses, adverse events and/or survival outcomes. These registries may not have population wide coverage in the strictest sense, but they do have a broad reach including private and public patients, and regional and metropolitan sites.

Like population-based observational research, our current projects are demonstrating that RRCTs can be conducted quickly and at low cost even with very large sample sizes. Long-term follow-up is also feasible, as the disease registries capture reliable data through to death. We argue that the reliability and depth of data eaptured in administrative databases will always be inferior to a dedicated disease registry, at least in part due to lack of context for the administrative databases. Of course, it is possible to tap into these administrative data sources to obtain or validate data for the disease registries. Indeed, we have and will continue to link with multiple other datasets within and outside the hospital to maintain data acouracy and completeness. ${ }^{5}$

As acknowledged by Tang et al. there are many known and unknown factors that confound treatment decision making and patient outcomes. In the absence of randomisation, any comparison of outcomes between different treatment groups is likely to be hypothesis generating only. RRCTs are an attempt to bridge the gap between conventional and observational trials, and while it may be difficult to match the quality of data captured in a conventional trial this is the reason for having a hard outcome, such as overall survival, as the primary endpoint of RRCTs.

Multiple RRCTs are now open and recruiting patients in Australia, further trials are in development across five tumour streams and extending beyond medical oncology. Significant progress has been made in engaging with several co-operative groups: e.g. the EX-TEM study, a randomised study exploring the duration of temozolomide therapy for glioblastoma patients, has now been endorsed by COGNO (Co-operative Trials Group for Neuro-Oncology) with 15 centres across Australia. Discussions have been initiated with several other co-operative groups regarding their endorsement of the RRCT model.

\section{Acknowledgments}

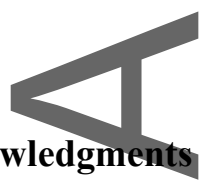

S.F. is supported by an Australian Government Research Training Program Scholarship. 


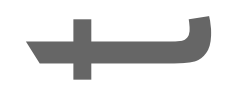

1 Tang M, Schaffer A, Pearson S-A. Embracing the full spectrum of real-world data for cancer medicines research in Australia. Asia Pac J Clin Oncol 2018.

2 Foroughi S, Wong H, Gately L, Lee M, Simons K, Tie J et al. Re-inventing the randomized controlled trial in medical oncology: The registry-based trial. Asia Pac J Clin Oncol 2018; 19.

3 Wachtell K, Lagerqvist B, Olivecrona GK, James SK, Fröbert O. Novel Trial Designs: Lessons Learned from Thrombus Aspiration During ST-Segment Elevation Myocardial Infarction in Scandinavia (TASTE) Trial. Curr Cardiol Rep 2016; 18: 11.

4 Field K, Wong H-L, Shapiro J, Kosmider S, Tie J, Bae S et al. Developing a national database for metastatic colorectal cancer management: perspectives and challenges. Intern Med J 2013; 43: 1224-1231.

5 Field K, Kosmider S, Johns J, Farrugia H, Hastie I, Croxford M et al. Linking data from hospital and cancer registry databases: Should this be standard practice? Intern Med J 2010; 40: $566-573$
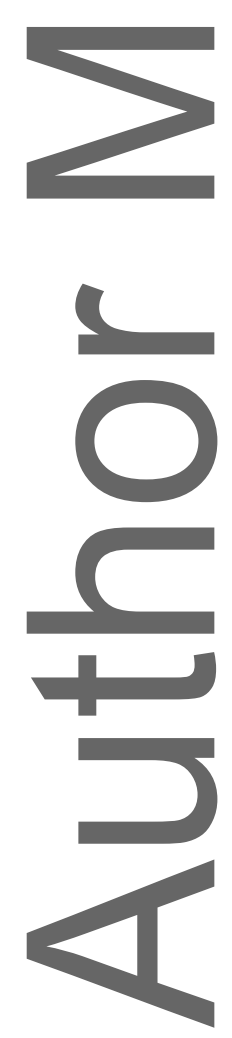


\section{University Library}

\section{- M M N E R VA A gateway to Melbourne's research publications}

Minerva Access is the Institutional Repository of The University of Melbourne

Author/s:

Foroughi, S;Wong, H-L;Gately, L;Lee, M;Simons, K;Tie, J;Burgess, AW;Gibbs, P

Title:

Registry-based randomized clinical trials as a method to improve cancer care in Australia

Date:

2019-06-01

Citation:

Foroughi, S., Wong, H. -L., Gately, L., Lee, M., Simons, K., Tie, J., Burgess, A. W. \& Gibbs, P. (2019). Registry-based randomized clinical trials as a method to improve cancer care in Australia. ASIA-PACIFIC JOURNAL OF CLINICAL ONCOLOGY, 15 (3), pp.188-189. https:// doi.org/10.1111/ajco.13122.

Persistent Link:

http://hdl.handle.net/11343/285443 